\title{
Le privilège d'une spécialité en mouvement
}

\section{The Privilege of a Specialty that moves}

\author{
J. Gligorov $\cdot$ S. Culine $\cdot$ I. Krakowski $\cdot$ P. Marti $\cdot$ J.-P. Metges $\cdot$ D. Serin $\cdot$ M. Schneider $\cdot$ M. Spielmann \\ (C) Springer-Verlag France 2014
}

Notre revue est heureuse et fière, en tant qu'organe de presse de formation continue en cancérologie, d'accueillir de nouveau notre institution et notre ministère de tutelle pour ce numéro dédié aux enjeux de la prise en charge actuelle et future des patients atteints de cancers.

Ces enjeux sont bien évidemment d'ordre scientifique, thérapeutique et organisationnel, mais ils deviennent également de plus en plus sociétaux, centrés sur des patients de plus en plus nombreux, vivant dans un monde de plus en plus difficile.

C'est dans ce contexte de politique de rigueur, avec des mutations démographiques importantes et des changements sociétaux profonds, que se pose un défi majeur : un accès équitable et de qualité pour tous à toutes les formes de soins des cancers y compris les plus innovants.

La création de l'Institut national du cancer en 2005 et les Plans cancer successifs ont indiscutablement permis d'accompagner et d'anticiper ces nouveaux enjeux [1-11]. Ils sont rappelés dans les articles portant sur l'amélioration des pratiques professionnelles, la personnalisation des parcours de soins et le soutien à l'innovation. En tant que professionnels de santé et acteurs de terrain de ces démarches, nous ne pou-

\section{J. Gligorov $(\bowtie)$}

AP-HP Tenon ; IUC-UPMC, F-75020 Paris, France

e-mail : joseph.gligorov@tnn.aphp.fr

S. Culine

Hôpital Saint-Louis, faculté Paris-VII, F-75010 Paris, France

I. Krakowski

Institut de cancérologie de Lorraine-Alexis-Vautrin,

F-54519 Vandœuvre-lès-Nancy, France

P. Marti

Centre hospitalier de la Côte-Basque,

F-64100 Bayonne, France

J.-P. Metges

CHU de Brest, hôpital Morvan, F-29609 Brest cedex, France

D. Serin

Institut Sainte-Catherine, F-84086 Avignon cedex 02, France

M. Schneider

Centre Antoine-Lacassagne, F-06189 Nice cedex 02, France

M. Spielmann

Gustave-Roussy, F-94805 Villejuif cedex, France vons que constater les progrès évidents apportés au quotidien dans ces domaines, mais il faut, dans un monde qui nous demande de nous évaluer à l'échelle nationale, le faire aussi à l'échelle internationale. La France fait partie du peloton de tête des pays industrialisés en ce qui concerne la proportion de patients guéris de leur cancer et l'espérance globale de vie des patients qui sont ou ont été traités pour un cancer [12].

Nous avons le privilège de bénéficier d'un système de santé bâti par nos prédécesseurs qui offre encore à ce jour l'accès à de nombreux outils diagnostiques, à des traitements spécifiques et des soins de support, soutiens sociaux qui font sa force et sa fragilité. Force oui, car comparé à d'autres pays, l'accès aux soins, aux plateformes de génétique moléculaire des cancers et aux médicaments est un atout, mais il est également une faiblesse car il ne se passe pas un jour sans que ne soient discutés les moyens qui, dans une logique comptable, sont des dépenses mais restent dans une logique de soins des investissements pour sauver des vies.

C'est dans une société qui évolue, mais l'évolution n'estelle pas par nature la caractéristique même du vivant, que nous devons répondre à de nouveaux défis liés entre autres aux données démographiques. En 2050, nous serons près de 70 millions, dont $25 \%$ de personnes âgées de plus de 65 ans et $15 \%$ de plus de 75 ans. L'espérance de vie augmente d'année en année, et cela pour toutes les catégories socioprofessionnelles, mais un ouvrier vit toujours six à sept ans de moins qu'un cadre supérieur (http://www.insee.fr).

Les fragilités sociales et médicales sont une source d'inégalité flagrante qui ponctue notre activité clinique en particulier dans les établissements accueillant les populations défavorisées qui ne sont pas que les sans-emploi, les personnes âgées, certaines populations migrantes, mais de plus en plus ce que nos sociologues appellent la " pauvreté laborieuse ", ceux pour qui la maladie avant d'être une angoisse de mort est une angoisse de misère [13-16].

Pour eux certes, il est essentiel de garantir l'accès à l'information, aux innovations, aux soins de qualité, mais le plus souvent il s'agit de répondre à des besoins vitaux et imminents qui sont parfois aussi simples que l'hébergement ou la nourriture. Comment, dans un système de tarification 
qui favorise la rentabilité technique à l'élaboration d'un projet de vie intégrant le soin et nécessitant du temps, répondre à ce défi ? Et comme il est encore plus frappant ce défi, en situation avancée de la maladie. À l'heure où notre société réfléchit à la légalisation de l'euthanasie active et au droit de mourir dans la dignité, certains souhaiteraient juste vivre les soins de leur cancer dans cette même dignité revendiquée.

La meilleure organisation indiscutable des soins en oncologie orchestrée par l'INCa depuis plusieurs années a également légitimement imposé de plus en plus de temps techniques d'évaluation, d'anticipation, d'accompagnement, d'explication, de soins, de suivi... Bref, de tâches professionnelles qui posent la question des nouveaux métiers du cancer et du transfert de compétences vers d'autres acteurs de soins qu'il faut valoriser également. Ces nouvelles organisations déjà initiées dans certains pays ont fait leur preuve comme l'a été l'apport des attachés de recherche clinique et techniciens d'études cliniques pour la recherche [17-21].

L'investissement dans la recherche reste l'une des garanties de la qualité de notre système de soins pour permettre l'accès aux innovations, mais à la vitesse à laquelle les évolutions technologiques progressent, les connaissances biologiques et l'information auprès des patients circulent, nous devons rester vigilants quant à la pertinence clinique de certains investissements académiques dont les retombées cliniques restent encore à ce jour limitées, ou tout du moins ont peu d'impact sur la prise en charge des patients comparativement à la promotion médiatique qui en est faite. Générer de l'espoir est fondamental dans notre profession et incombe légitimement à ceux qui sont à la tête de ces programmes, mais gérer la déception lorsque l'espoir a pu être ressenti comme la dernière chance incombe parfois à d'autres. Nous avons le privilège d'exercer une profession et une spécialité à la croisée des enjeux sociétaux, scientifiques, bref humains ; ce sont toutes ses composantes qu'il nous faut intégrer, et c'est l'objet de ce numéro de l'Institut national du cancer.

\section{Références}

1. Bôquet R, Chantôme G (2014) Le dispositif d'autorisation du traitement du cancer (in partie I : Les apports des Plans cancer au service de l'amélioration des pratiques professionnelles). Oncologie 16: HS11-13

2. Desaulle MS (2014) De l'intérêt pour une agence régionale de santé d'un réseau régional en cancérologie : structure régionale d'appui et d'expertise en cancérologie générale, pédiatrique et oncogériatrie (in partie I : Les apports des Plans cancer au service de l'amélioration des pratiques professionnelles). Oncologie 16: HS14-15

3. Bréchot JM (2014) L'organisation spécifique en oncogériatrie, le point de vue institutionnel (in partie I : Les apports des Plans cancer au service de l'amélioration des pratiques professionnelles). Oncologie 16: HS16-18

4. Kurtz JE, Heitz D (2014) Les apports des Plans cancer dans l'amélioration des pratiques professionnelles en oncogériatrie : le point de vue des professionnels (in partie I : Les apports des
Plans cancer au service de l'amélioration des pratiques professionnelles). Oncologie 16: HS19-20

5. Verdoni L, Mazeau-Woynar V (2014) Enjeux et principes d'une coordination nationale des acteurs pour la production, l'implémentation et l'évaluation des outils d'aides à la décision médicale en cancérologie (in partie I : Les apports des Plans cancer au service de l'amélioration des pratiques professionnelles). Oncologie 16: HS21-25

6. Pourcel G (2014) Le parcours en cancérologie : vers un modèle de prise en charge intégrée et cordonnée (in partie II : Un parcours personnalisé et coordonné pour mieux répondre aux besoins des patients). Oncologie 16: HS26-28

7. Hans S (2014) Exemple du parcours personnalisé des patients atteints de cancer ORL : une vision pluriprofessionnelle de la prise en charge (in partie II : Un parcours personnalisé et coordonné pour mieux répondre aux besoins des patients). Oncologie 16: HS29-31

8. Duperray M, Mazeau-Woynar V (2014) La plateforme Cancer info : une réponse au besoin d'information des patients et de leurs proches (in partie II : Un parcours personnalisé et coordonné pour mieux répondre aux besoins des patients). Oncologie 16: HS32-34

9. Merlet F, Hoog-Labouret N (2014) Intégrer la préservation de la fertilité dans le parcours de soins en cancérologie (in partie II : Un parcours personnalisé et coordonné pour mieux répondre aux besoins des patients). Oncologie 16: HS35-38

10. Ancellin R, Bessette D, Chauvet C, et al. (2014) La prévention des cancers : rôle et implication des professionnels de santé (in partie III : Les nouveaux enjeux de la prévention et du dépistage). Oncologie 16: HS39-44

11. de Bels F, Viguier J (2014) Cancer du sein et du cancer colorectal : proposer à chaque personne la modalité de dépistage ou de suivi adaptée à son niveau de risque (in partie III : Les nouveaux enjeux de la prévention et du dépistage). Oncologie 16: HS45-47

12. De Angelis R, Sant M, Coleman MP, et al. and EUROCARE-5 Working Group (2014) Cancer survival in Europe 1999-2007 by country and age: results of EUROCARE-5 - a population-based study. Lancet Oncol 15: 23-34

13. Lafay L, Bousquet PJ, Viguier J (2014) Piloter la politique de lutte contre le cancer sur des données robustes et partagées (partie V). Oncologie 16: HS69-71

14. Vandewalle H, Affeltranger B, Bungener C (2014) Inégalités de santé : lutter à tous les temps du cancer (partie VI). Oncologie 16: HS72-74

15. Godet J (2014) Comment, selon la Ligue contre le cancer, le Plan cancer III pourrait conjuguer ses objectifs de santé publique et l'impérieuse nécessité de faire vivre plus fortement la démocratie sanitaire ? (in partie VII : Démocratie sanitaire). Oncologie 16: HS75-76

16. Lambert M (2014) Quand le Plan cancer participe d'une démocratie sanitaire comme troisième pilier de la Stratégie nationale de santé (in partie VII : Démocratie sanitaire). Oncologie 16: HS77-78

17. Bachouche N, Bussière B, Hommais A, et al. (2014) L'organisation et le soutien à la recherche clinique publique en cancérologie (in partie IV : Soutenir l'innovation scientifique en cancérologie). Oncologie 16: HS48-57

18. Nowak F, Calvo F (2014) Les enjeux pour le développement de la médecine personnalisée (in partie IV : L'organisation et le soutien à la recherche clinique). Oncologie 16: HS58-60

19. Vassal G (2014) AcSé crizotinib, un essai pionnier du programme AcSé dans le champ de la médecine personnalisée (in partie IV : L'organisation et le soutien à la recherche clinique). Oncologie 16: HS61-62

20. Gerbaud E, Gomez S (2014) La formation à la recherche translationnelle : le cancer comme modèle de recherche multidisciplinaire (in partie IV : L'organisation et le soutien à la recherche clinique). Oncologie 16: HS63-66

21. Raynaud J, Dufour F (2014) Fondation ARC : un catalyseur pour de nouvelles solutions thérapeutiques (in partie IV : L'organisation et le soutien à la recherche clinique). Oncologie 16: HS67-68 